This item was submitted to Loughborough's Research Repository by the author.

Items in Figshare are protected by copyright, with all rights reserved, unless otherwise indicated.

\title{
Priming to promote fluent motor skill execution: Exploring attentional demands
}

PLEASE CITE THE PUBLISHED VERSION

https://doi.org/10.1123/jsep.2013-0085

\section{PUBLISHER}

(c) Human Kinetics, Inc.

\section{VERSION}

AM (Accepted Manuscript)

\section{PUBLISHER STATEMENT}

This work is made available according to the conditions of the Creative Commons Attribution-NonCommercialNoDerivatives 4.0 International (CC BY-NC-ND 4.0) licence. Full details of this licence are available at: https://creativecommons.org/licenses/by-nc-nd/4.0/

\section{LICENCE}

CC BY-NC-ND 4.0

\section{REPOSITORY RECORD}

Adams, Danielle, Kelly J. Ashford, and Robin C. Jackson. 2019. "Priming to Promote Fluent Motor Skill Execution: Exploring Attentional Demands". figshare. https://hdl.handle.net/2134/35521. 
1 Running head: The Efficacy of Priming to Promote Fluent Motor Skill Execution

2

3

4

5

6

7

8

Priming to Promote Fluent Motor Skill Execution: Exploring Attentional Demands

9

10

11

12

13

14

15

16

17

18

19

20

21

22

23

24

25 Submitted: $26 / 04 / 13$

26 
28 The effect of priming on the speed and accuracy of skilled performance and on a probe

29 reaction time task designed to measure residual attentional capacity, was assessed. Twenty-

30 four skilled soccer players completed a dribbling task under three prime conditions (fluency,

31 skill-focus and neutral) and a control condition. Results revealed changes in trial completion

32 time and secondary task performance in line with successful priming autonomous and skill-

33 focused attention. Retention test data for task completion time and probe reaction time

34 indicated a linear decrease in the priming effect such that the effect was non-significant after

3530 minutes. Results provide further support for the efficacy of priming and provide the first

36 evidence of concurrent changes in attentional demands, consistent with promoting or

37 disrupting automatic skill execution.

38

39 Keywords: conscious processing, priming, P-RT, automatic control.

40 


\section{Introduction}

42 Attentional processes have been identified as significant mediators of expert motor skill execution (Beilock, Carr, McMahon, \& Starkes, 2002; Beilock, Wierenga, \& Carr, 2002;

44 Beilock, Bertenthal, McCoy, \& Carr, 2004; Hardy, Mullen, \& Martin, 2001; Wulf, McNevin, 45 \& Shea, 2001; Zachry, Wulf, Mercer, \& Bezodis, 2005). In skilled performers, the 46 detrimental effect of focusing attention internally has been widely documented in motor and 47 perceptual-motor tasks (e.g., Beilock \& Carr, 2001; Bell \& Hardy, 2009; Gray, 2004; Hardy, Mullen, \& Jones, 1996; Jackson, Ashford, \& Norsworthy, 2006). A widely accepted explanation is that the components of the skill performed by an expert have become

50 proceduralized in long-term memory (Fitts \& Posner, 1967) thus run under reduced levels of conscious control (i.e., more automatically). By refocusing attention on those proceduralized components, skill processes are brought back into working memory, and decomposed into smaller units (Masters \& Maxwell, 2008) resulting in a decrement in performance.

Researchers have explored ways of optimizing attentional focus of expert performers in order to promote automated performance. These interventions include the use of multicomponent interventions embedded in pre-performance routines (Mesagno, Marchant, \& Morris, 2008; Mesagno \& Mullane-Grant, 2010), the use of concurrent secondary tasks (Beilock, Carr et al., 2002; Beilock, Wierenga et al., 2002; Gray, 2004), adopting strategies that promote an external focus of attention (Wulf, 2013), and visual attention training (Vine,

60 Moore, \& Wilson, 2011). While each of these methods has shown promise, there are associated practical and theoretical limitations. For example, multi-component preperformance interventions make it difficult to determine the source(s) of any improvements although insight can be gained from retrospective verbal reports. Concurrent secondary tasks

64 are more targeted towards different aspects of working memory and associated attentional

65 resources; however, there are contradictory findings in the literature. For example, dual-task 
conditions were found to facilitate performance in skilled golfers and experienced soccer players (Beilock, Carr et al.) and random letter generation was found to increase golf putting accuracy under high anxiety (Mullen \& Hardy, 2000). Conversely, Mullen, Hardy, and Tattersall (2005) found that experienced golfers putted more poorly under high anxiety than low anxiety when performing a concurrent secondary tone counting task.

\section{Priming}

Another potential method of promoting fluent, effortless performance is through the use of priming. The term 'priming' is used to describe "the influence a stimulus has on subsequent performance of the processing system" (Baddeley, 1997, p. 352). Through the activation of specific contexts, traits, stereotypes, goals and related constructs, priming is hypothesized to stimulate the representations of behaviors that influence a general behavioral change in line with those representations (Bargh, Chen, \& Burrows, 1996; Chen, \& Bargh, 1997; Dijksterhuis \& Van Knippenberg, 1998). Priming was traditionally utilised to explore the relative automaticity of certain behaviours and has since developed into the investigation of the manipulation or activation of desired behaviours unconsciously through priming methods (see Bargh \& Chartrand, 2000). Specifically, once stimulated, changes in perception, evaluations, motivation or social behavior have been observed (see Dijksterhuis \& Bargh, 2001; Wheeler \& Petty, 2001, for reviews).

While many studies support the efficacy of priming in the afore-mentioned behavioural categories, there is a paucity of research examining the effect of priming on skilled motor behavior and the underlying processes that mediate any observed effects. This area warrants further study considering the benefits of unconscious control of expert motor skill execution and the principles of priming research. In early studies of priming (Bargh et al., 1996 - Experiment 2; Hull, Stone, Meteyer, \& Matthews, 2002 - Experiments 1a \& 1b) researchers found that priming participants with an elderly stereotype resulted in slower 
91 walking in both elderly and young college students. Further, Macrae et al., (1998)

92 demonstrated that priming participants with the notion of a world champion racing driver

93 resulted in faster walking. Similarly, Aarts and Dijksterhuis (2002) found that priming

94 participants with words associated with fast animals (cheetah, antelope) or slow animals

95 (snail, turtle) led to faster and slower walking speeds, respectively. In relation to skilled motor behavior, Bry, Meyer, Oberlé, and Gherson (2009) found improved relay changeover speed in beginner track athletes through priming cooperation, where cooperation was considered as the adaptation of one's behaviour to suit another's in the pursuit of a collective goal, while Stone, Lynch, Sjomeling, and Darley (1999) found decrements in golf putting

100 performance following activation of a racial stereotype prime.

\section{Priming to Promote Fluent Motor Skill Execution}

The rationale underpinning priming research is that automatic processes can be instigated by environmental triggers (Bargh \& Chartrand, 2002). Extending this idea to the sport domain, Ashford and Jackson (2010) examined the effect of priming in a group of skilled field-hockey players performing a dribbling task under low and high pressure. In two experiments, a positive prime containing target words relating to the concept of automaticity resulted in significantly faster and more accurate performance than that attained in the control condition (Experiments $1 \& 2$ ) and negative or neutral prime conditions (Experiment 2).

109 Conversely, the negative prime resulted in significantly slower performance than the neutral 110 prime.

Ashford and Jackson (2010) interpreted their results by appealing to attentional

112 mediators of performance. In-line with self-focus theories (Baumeister, 1984; Masters, 1992)

113 they suggested that the positive and negative primes may have successfully directed attention

114 away from and towards the mechanics of movement execution, respectively. While plausible,

115 this interpretation requires confirmation through measuring changes in the attentional 
116 demands associated with performance following priming. More fundamentally, in a careful

117 replication of the protocol used in Ashford and Jackson's study, Winter and Collins (2013)

118 found no difference between the control condition and a prime condition designed to promote

119 autonomous performance. Participant performance in the PETTLEP imagery protocol

120 condition was better than both the priming and control conditions, calling into question the

121 robustness of the priming effect.

122 The Retention of Primed Behaviors

123 A number of studies indicate that the behavioral effects of cognitive priming are strongest

124 immediately following exposure to the prime (Bargh, 1997; Bargh \& Gollwitzer, 1994) and

125 significantly attenuate after approximately five minutes (e.g., Bargh, Gollwitzer, Lee-Chai,

126 Barndollar, \& Trötschel, 2001; Bargh, Lombardi, \& Higgins, 1988; Higgins, Bargh, \&

127 Lombardi, 1985). The longer-term retention of behavioral effects resulting from

128 unconsciously perceived stimuli is unconfirmed. Merikle and Daneman (1998) noted that the

129 majority of priming studies had tested for primed effects within five minutes of exposure and

130 had not explicitly examined subsequent retention of observed effects. Based on a meta-

131 analysis of studies investigating memory for events during general anaesthesia (Merikle \&

132 Daneman, 1996), they proposed that unconsciously perceived stimuli can last for many hours.

133 In addition, Srull and Wyer (1979) showed that priming hostility can impact social judgments

134 up to one week after the priming period, when the to-be-judged stimulus had been presented

135 right after the priming event, yet the priming effect was not retained one week later when

136 exposure to the stimulus was also delayed. Bargh et al. (2001), proposed that effects

137 exceeding the 4 to 5 minute timeframe result indirectly from psychological mediators

138 stemming from the behavioural consequences of priming rather than directly from the

139 priming intervention. For example, Nelson and Norton (2005) found that participants

140 demonstrated an increased willingness to complete volunteer work three months after they 
141 were primed with the category 'superhero'. It is possible that this increased willingness over

142 an extended period was mediated by the satisfaction gained through the act of helping (Bargh

143 et al., 2001). In the domain of skilled motor behavior the durability of any priming effect is

144 presently unknown and is clearly an important consideration given the large range in duration

145 of competitive sport activities.

\section{The Present Study}

147 The present study addressed three specific aims in investigating the efficacy of

148 priming on motor skill behavior. The first aim was to replicate the findings of Ashford and

149 Jackson (2010), who reported content-related changes in motor performance following

150 fluency priming and skill-focus priming interventions. The second aim was to investigate the

151 attentional demands associated with motor performance under the different prime conditions.

152 In movement-related research, probe-reaction time (P-RT) tasks have been used to assess the

153 'mental workload' imposed on the performer by any particular set of task conditions

154 (Abernethy, 1988). P-RT task performance is considered to be a reflection of residual

155 processing capacity, with performance being proportional to the size of the remaining 'free'

156 attentional space (e.g., Wulf et al., 2001). Faster responses to the secondary P-RT task are

157 interpreted to indicate that less on-line attention was utilized for primary task performance. In

158 the present study, inclusion of the P-RT task allowed for assessment of the relative

159 automaticity of motor skill execution as a function of the priming conditions. Based on the

160 premise that the priming interventions promote or disrupt automatic motor processes through

161 reducing or increasing conscious control, we hypothesized that a fluency based prime would

162 yield faster P-RTs than a skill-focus prime, neutral prime and a no-prime control.

163 Accordingly, we hypothesized that a skill-focus prime would yield slower P-RTs than the

164 neutral prime and control conditions. 

absence of any data pertaining to this issue in skilled motor behavior, and considering the varying lengths of sport competitions the final aim was to explore the retention of priming

168 effects over a one hour period.

\section{Method}

\section{$170 \quad$ Participants}

After gaining institutional ethical approval, 24 skilled male soccer players, aged between 18 and 21 years $(M=19.2 ; S D=0.9)$, provided informed consent to participate in

173 the study. Participants were members of a university first or second teams, currently

174 competing in university league matches and reported a mean of 12.8 years $(S D=2.9$ years $)$ of

175 involvement in organized, competitive soccer. The number of participants was selected after conducting a power analysis (G-power version 3.1) entering a medium affect size $(f=0.25)$, power set at 0.80 and a correlation of 0.5 among the repeated measures. This generated a

178 sample size of $n=24$ yielding power of 0.82 . In addition, 24 participants allowed for

179 complete counterbalancing of the experimental conditions.

\section{Task and Apparatus}

\section{Soccer dribbling task}

The primary task required participants to dribble a standard size soccer ball through a series of six cones spaced at $1.5 \mathrm{~m}$ intervals using the in-step and out-step of their dominant

184 foot (Beilock, Carr et al., 2002; Jackson et al., 2006). Participants were instructed to complete the trials as quickly and accurately as possible and were informed that task completion time and the accuracy of their dribbling would be recorded. Newtest Power Timer 1.0

187 photoelectric cells were placed at the start and finish to record trial completion time to the 188 nearest millisecond. 
190 grid adjacent to each cone comprised five vertical lines drawn in parallel to the midline of the

191 course, spaced $5 \mathrm{~cm}$ apart, with the first line drawn $10 \mathrm{~cm}$ from the midline. A concealed

192 digital video camera (Panasonic NVDS65B), was positioned at the end of the course to

193 record each trial. Subsequently, the maximum displacement of the ball in the grid adjacent to

194 each cone was determined from the video recordings and mean values then calculated for

195 each prime condition. In addition, $10 \%$ of trials in each condition were randomly selected and

196 assessed by an independent rater.

197 Probe-reaction task (P-RT)

198 In the secondary task participants were instructed to respond as quickly and accurately 199 as possible to an auditory stimulus of $80 \mathrm{~ms}$ duration (Gray, 2004). The randomly presented 200 tone had a frequency of either $250 \mathrm{~Hz}$ or $500 \mathrm{~Hz}$ and participants were instructed to identify it 201 as either 'low' or 'high'. The participant's responses were recorded by a digital voice recorder

202 (Olympus model DS-50) affixed to the participant's waist via a small microphone clipped on 203 to the neck-line of the participant's clothing...Subsequently, P-RT was determined from the 204 visual representation of the amplitude and frequency of the tone and vocal response using 205 Wavelab 6.1.1.

\section{Conditions}

Participants completed the task under three priming conditions (fluency, skill-focus,

208 and neutral), each of which took the form of a scrambled sentence task (Bargh et al., 1996;

209 Hull et al., 2002; Srull \& Wyer, 1979). The scrambled sentence tasks were those used by

210 Ashford and Jackson (2010). Each comprised 30 items, consisting of five words per item

211 presented in random order, four of which could be used to form a sentence. Participants were

212 instructed to use four of the five words to form a grammatically correct sentence and to write 
213 out the whole sentence in a space provide below the randomly presented words. Items for

214 each of the three priming tasks had been previously assessed for face validity by two experts.

215 Fluency prime. Target words were based on literature relating to the concepts of 216 automaticity, optimal performance, and flow; for example, 'movements seemed to flow' and

217 'I am at ease' (presented as: 'movements very flow to seemed' and 'am I ease at on').

218 Skill-focused prime. Target words were drawn from research on attentional focus

219 and conscious control and directed the performer to the execution of the skill; for example, 'I

220 focused on technique' and 'hip position is important' (presented as: 'technique on I the

221 focused' and 'important position is hip correct,' respectively).

222 Neutral prime. Target words bore no relation to performance; for example, 'the grass

223 is green' and 'the world is round' (presented as: 'green is purple grass the' and 'square round

224 the is world,' respectively).

225 Control. In the control condition, participants were simply instructed to "complete

226 the dribbling task as quickly and accurately as possible”.

227 Procedure

228 A repeated measures design was employed in which conditions were fully

229 counterbalanced. Prior to the test trials, participants performed 10 familiarization trials. A

230 total of six blocks (three priming, one control, two retention) of five test trials followed.

231 Participants were given time between trials for their breathing rate to return to normal. A

232 block of 5 trials took approximately 4 minutes in total. Participants responded to a single

233 auditory stimulus in four of the five trials in each block. A single trial without the auditory

234 stimulus occurred randomly within each block to allow for testing of the impact of the

235 secondary task on primary task performance. Prior to each priming block, participants

236 completed a scrambled sentence task appropriate to the particular condition. In line with Hull

237 et al. (2002), participants were advised that this grammatical task was part of an unrelated 
research project and were asked if they could complete it during their rest period. With the exception of the last block of trials participants were given a short rest period of 2-3 minutes

240 after each block, during which they were requested to count backwards in sevens from 70 .

241 This working memory intensive task was included to prevent rumination about performance

242 in the previous block of trials and to decrease the accessibility of the previous concept.

After completing the priming and control conditions participants were given a 30-

244 minute break. Participants then completed the first block of retention trials after which they were given an additional 30-minute break before completing the second block of retention trials. During these breaks, participants were asked not to discuss the study with anyone and to refrain from soccer dribbling. Upon completion of the experiment, each participant was shown the camera recording ball displacement and asked for their consent to use the video footage for analysis. Finally, each participant was thanked for participating, was debriefed about the purpose of the study, and was requested not to discuss the specific purpose of the study with other potential participants.

\section{Data Analysis}

Prior to analysis, all data were screened for outliers using standardized scores $(z \pm$ 3.29) and the Mahalanobis distance test. Initially, to test whether the secondary task impacted primary task performance, a 2 (with/without P-RT) x 4 (prime condition) repeated measures ANOVA was conducted. Following this, one-way repeated measures ANOVAs were conducted for the performance data with prime condition entered as a within-participants factor and task completion time, lateral displacement and P-RT serving as the dependent variables.

In order to analyze the retention data while retaining statistical power, the participant sample was divided into two groups according to the last condition they completed. The

262 control group $(n=12)$ comprised participants who had completed the neutral or control 
condition as their last block while the experimental group $(n=12)$ comprised participants

264 who completed the fluency or skill-focus priming conditions last. As retention of a priming 265 effect in participants exposed to the fluency and skill primes would result in opposite effects

266 on performance, retention scores for participants who received the skill-focus prime last were

267 reversed such that negative scores indicate the presence of a priming effect. The three

268 difference scores (task completion time, lateral displacement, P-RT) were calculated relative to performance in the control condition and depict the presence / absence of a priming effect immediately after exposure to the last prime (baseline) and at the 30-minute and 60-minute retention tests. To analyse these data, one-sample $t$-test comparisons (one-tailed) were made against a value of 0 at each of the three retention points.

\section{Results}

\section{Initial Effects of Prime}

275 Data screening revealed no univariate or multivariate outliers. Further analyses confirmed

276 that P-RT had no impact on primary task performance (see Footnote ${ }^{1}$ ) and that the prime

277 effect remained constant across trial blocks (see Footnote ${ }^{2}$ ).

Task completion time. A repeated measures one-way ANOVA for task completion time revealed a significant main effect of priming condition, $F(3,21)=30.01, p<.001, \eta_{p}{ }^{2}$

$280=.81$. Follow-up pairwise comparisons with Bonferroni adjustment revealed task completion

281 time to be significantly faster in the fluency prime condition than in the neutral prime, and control conditions $(p<.001)$. Additionally, task completion time in the skill-focused prime condition was significantly slower than in the neutral prime and control conditions $(p<.001)$

284 (Figure 1, top panel).

Lateral Displacement. A repeated measures one-way ANOVA for lateral

286 displacement revealed a non-significant main effect of prime condition, $F(3,21)=1.50, p=$ 287 $.24, \eta_{p}^{2}=.18$ (Figure 1, middle panel). 
effect of prime condition, $F(3,21)=7.39, p<.001, \eta_{p}{ }^{2}=.51$. Follow-up pairwise comparisons with Bonferroni adjustment revealed P-RT to be significantly faster in the

291 fluency prime condition than in the skill focus prime condition $(p=.001)$. Comparisons of each of these conditions with the neutral prime and control conditions revealed no significant differences (Figure 1, bottom panel).

\section{Retention of Primed Behaviors}

As can be seen in Figure 1 (right panels, experimental group), there was a linear attenuation of the priming effect for both completion time and P-RT data across the baseline, 30-minute and 60-minute retention test points. As expected, the control group's performance remained relatively stable across the retention points.

Task completion time. The one-sample $t$-tests revealed non-significant effects for the control group at baseline $(p=.22)$, and in the 30-minute $(p=.23)$ and 60 -minute $(p=.18)$ retention tests. For the experimental group the effect of the prime was significant at baseline $(p=.02)$ but was non-significant at both the 30-minute $(p=.10)$ and 60 -minute $(p=.40)$ retention tests. displacement revealed non-significant effects at baseline $(p=.17)$, and at the 30 -minute $(p=$ .12) retention test, and, unexpectedly, a significant difference at the 60 -minute $(p=.03)$ retention test. For the experimental group the effect of the prime on lateral displacement was non-significant at baseline $(p=.32)$ and at both the 30-minute $(p=.10)$ and 60-minute $(p=$ .09) retention tests.

P-RT. The one-sample $t$-tests for probe reaction time revealed non-significant effects

311 for the control group at baseline $(p=.28)$, and in the 30-minute $(p=.46)$ and 60-minute $(p=$ $312.30)$ retention tests. For the experimental group the effect of the prime was significant at 
313 baseline $(p=.03)$ but was non-significant at both the 30-minute $(p=.12)$ and 60 -minute $(p=$

$314 \quad .19)$ retention tests.

\section{Discussion}

The aim of the present study was to further investigate the efficacy of priming in

317 skilled motor behavior, utilizing a sentence scrambling task. In particular, we aimed to assess

318 differential use of attentional resources following different primes by assessing the speed and accuracy of a soccer skill as well as response to a P-RT task. Participants were assessed under conditions designed to optimize performance by priming words relating to fluent execution and to hinder performance by priming words associated with conscious control. Finally, we sought to explore the retention of priming effects over a one hour period.

The results provide support for the viability of priming in influencing skilled motor

behavior. Following exposure to the task designed to prime autonomous, fluent execution, task completion time was significantly faster than in the control condition. By contrast, following the skill-focus prime, task completion time was significantly slower than in the control condition. Importantly, the present findings provide support for the conscious processing hypothesis and research concerning reinvestment (e.g., Baumeister, 1984; 2000). Specifically, predicted changes in P-RT mirrored changes in performance such that PRT was significantly faster following the fluency prime than after the skill-focus prime. The performance effects observed in this study replicate the findings of Ashford and

333 Jackson (2010) but conflict with the null effects reported by Winter and Collins (2013). It is

334 presently unclear why similar protocols, tasks, participants and measures have resulted in such different findings. One possibility is that there was greater scope for improving

336 performance in Ashford and Jackson's study than there was in Winter and Collins' study. The

337 participants in Winter and Collins' study were older, more experienced and competed at a 
higher level (from county to international level) and had faster mean trial completion times than those in Ashford and Jackson's study. By definition, the extent to which priming the concept of fluent autonomous performance will impact participants is dependent on the preexisting level of fluency or automaticity. Cross-sectional designs examining the efficacy of priming in groups of varying experience and ability will help address this question.

344 other studies using the sentence completion task (REFs) and might be explained by the contextual overlap and self-relevant nature of the prime. When a prime is aligned with an individual's self-concept, a subconscious comparison process is activated resulting in behavior modification (Bruce, Carton, Burton, \& Ellis, 2000; Hull et al., 2002). With respect to this contextual overlap, conscious control is the main characteristic of the beginning/cognitive stage of learning while automaticity is the main characteristic of an expert (Fitts \& Posner, 1967). Consequently, the concepts used in the priming task were likely to relate to each participant in the study as well as to skill execution in the task itself. In addition, Hull et al (2002) found that people were more sensitive to self-relevant primes and that the effects of self-relevant primes are sometimes easier to obtain. and Dimmock (2008) who discussed how performance patterns can be attributed to the content of conscious processing and the influence of this on generalised motor schema.

357 Gucciardi and Dimmock argued that global thoughts (i.e., swing thoughts or, in the case of

358 the present study, fluency primes) promote selection of an appropriate motor program as the thoughts provide a holistic representation of the skill. This type of global processing would

360 appear to demand few attentional resources as did performance following the fluency prime 361 in the present study, evidenced by faster P-RTs. In contrast, explicit cues that focus on the 362 technical components of a skill place greater demands on working memory and attentional 
363 resources as did performance following the skill-focus prime in the present study, evidenced

364 by increased P-RTs. The extent to which priming and using verbal cues represent different

365 means of achieving functionally equivalent outcomes is yet to be determined.

366 Analysis of the lateral displacement data confirmed that changes in task completion

367 time were not at the expense of dribbling accuracy. This finding differs slightly from the

368 results of Ashford and Jackson (2010) who found that both performance speed and lateral

369 displacement were affected with improvements and decrements in performance observed in

370 the positive and negative prime conditions, respectively. As lateral displacement was largely

371 unaffected by priming in the present study, differences in task completion time are likely to

372 have been caused by differences in sequencing and timing of motor responses, which have

373 been shown to change as a function of attentional focus in various tasks (Beilock \& Carr,

374 2001; Collins, Jones, Fairweather, Doolan, \& Priestly, 2001, Gray, 2004). In future,

375 kinematic analysis would enable researchers to pinpoint the precise spatiotemporal

376 parameters underpinning changes in task completion time resulting from priming (Gray,

377 2004; Pijpers, Oudejans, Holsheimer, \& Bakker, 2005).

378 With reference to the exploratory analysis examining the retention of the primed

379 behavioural effects, a linear attenuation of the priming effect was observed with respect to

380 completion time and P-RT, such that the effect was non-significant after 30 minutes and

381 entirely absent after one hour. This is broadly consistent with priming research in other

382 domains, which has shown significant attenuation of priming effects after just five minutes

383 (e.g., Bargh et al., 1988; Bargh et al., 2001; Higgins et al., 1985). Set against this, analysis of

384 performance across the trials within each condition in the present study revealed a non-

385 significant effect of trial number. Each block of trials took approximately four minutes to

386 complete suggesting the priming effect was retained for at least four minutes. Given the

387 relatively small number of participants in each group $(n=12)$ for this analysis and the fact 
that retention of the priming effect was tested across two relatively large 30-minute time

windows, a more systematic examination of the attenuation of the priming effect is warranted.

While the findings extend those of Ashford and Jackson (2010) further limitations of the present study should be acknowledged. First, owing to the nature of the experimental setting, the ecological validity of the task can be questioned. While the soccer dribbling task is representative of a skill-based drill conducted in training and a technique used within a game situation, it is important that the efficacy of techniques established in the laboratory, is assessed in field settings to confirm their effectiveness and robustness (Tipper \& Weaver, 1998). Second, while the efficacy of the priming intervention was supported, the process was not necessarily implicit. The task was introduced to participants as an additional and unrelated task in line with instructions given to participants in previous studies (e.g., Srull \& Wyer, 1979, Hull et al., 2002), yet expectations about a link between the priming task and the subsequent motor task may have been formed. While informal questioning of the participants after the experiment did not reveal evidence of participants making a connection between the priming task and the motor task a more sensitive formal assessment of participants' awareness of, and hypotheses about, the link between the priming task and its effect on the 'unrelated' performance task is warranted. This will also help determine whether the priming paradigm invokes use of target words in a functionally equivalent manner to the more overt or explicit use of cue words (Gucciardi \& Dimmock, 2008; Mullen \& Hardy, 2010). skilled motor behavior. Importantly, the analysis revealed differences in the attentional

410 demands associated with performance that were consistent with the nature of the primes and

411 observed performance: priming fluency enhanced motor performance and was associated 412 with faster P-RTs, while priming skill focus was detrimental to performance and was 
413 associated with slower P-RTs. With research applying priming to skilled motor behaviour in

414 its infancy and already subject to conflicting findings, the robustness of the phenomenon

415 needs to be established across different sporting activities. A logical extension to the present

416 study is to determine whether the observed priming effects are moderated by either skill level

417 or participant awareness of the link between the priming and motor tasks. In so doing, the

418 processes through which priming impacts skilled performance will be better understood. The

419 extent to which priming can influence psychological factors impacting sports performance,

420 the robustness of primed effects over time, and the degree of transfer of primed effects to the

421 field, offer additional theoretical and practical avenues for research. 
424 Aarts, H., \& Dijksterhuis, A. (2002). Category activation effects in judgement and behaviour: The moderating role of perceived comparability. British Journal of Social Psychology, 41, 123-138.

Abernethy, B. (1988). Dual-task methodology and motor skills research: Some application and methodological constraints. Journal of Human Movement Studies, 14, 101-132.

Ashford, K.J., \& Jackson, R.C. (2010). Priming as a means of preventing skill failure under pressure. Journal of Sport and Exercise Psychology, 32, 518-536.

Baddeley, A. (1997). Human memory: Theory and practice. Hove, UK: psychology Press.

Bargh, J.A. (1997). The automaticity of everyday life. In R. S. Wyer, Jr. (Ed.), The Mahwah, NJ: Erlbaum.

Bargh, J.A., \& Chartrand, T.L. (2000). The mind in the middle: A practical guide to priming and automaticity research. In H.T. Reis \& C.M. Judd (Eds.), Handbook of research methods in social and personality psychology (pp. 253-285). New York: Cambridge University Press.

Bargh, J.A., Chen, M., \& Burrows, L. (1996). Automaticity of social behaviour: Direct effects of trait construct and stereotype activation on action. Journal of Personality and Social Psychology, 71, 230-244.

442 Bargh, J.A., \& Gollwitzer, P.M. (1994). Environmental control of goal-directed action: Automatic and strategic contingencies between situations and behaviour. In W. Spaulding (Ed.), Nebraska symposium on Motivation (Vol, 41, pp. 71-124). Lincoln: University of Nebraska Press. 
Bargh, J.A., Gollwitzer, P.M., Lee-Chai, A., Barndollar, K., \& Trötschel, R. (2001). The automated will: Nonconscious activation and pursuit of behavioural goals. Journal of Personality and Social Psychology, 81, 1014-1027.

Bargh, J.A., Lombardi, W.J., \& Higgins, E.T. (1988). Automaticity of chronically accessible constructs in person X situation effects on person perception: It's just a matter of time. Journal of Personality and Social Psychology, 55, 599-605.

Baumeister, R. F. (1984). Choking under pressure: Self-consciousness and paradoxical effects of incentives in skilful performance. Journal of Personality and Social Psychology, 46, 610-620.

Beilock, S.L., Bertenthal, B.I., McCoy, A.M., \& Carr, T.H. (2004). Haste does not always make waste: Expertise, direction of attention, and speed versus accuracy in performing sensorimotor skills. Psychonomic Bulletin and Review, 11, 373-379.

Beilock, S.L., \& Carr, T.H. (2001). On the fragility of skilled performance: What governs choking under pressure? Journal of Experimental Psychology: General, 130, 701-725.

Beilock, S.L., Carr, T.H., McMahon, C., \& Starkes, J.L. (2002). When paying attention becomes counterproductive: Impact of divided attention versus skill-focused attention on novice and experimental performance of sensorimotor skills. Journal of Experimental Psychology: Applied, 8, 6-16.

Beilock, S.L., Wierenga, S.A., \& Carr, T.H. (2002). Expertise, attention, and memory in sensorimotor skill execution: Impact of novel task constraints on dual-task performance and episodic-memory. The Quarterly Journal of Experimental Psychology, 55, 1211-1240.

Bell, J. J., \& Hardy, J. (2009). Effects of attentional focus on skilled performance in golf. Journal of Applied Sport Psychology, 21, 163-177. 
Bruce, V., Carson, D., Burton, M.A., \& Ellis, A.W. (2000). Perceptual priming is not a necessary consequence of semantic classification of pictures. The Quarterly Journal of Experimental Psychology, 53, 289-323

Bry, C., Meyer, T., Oberlé, D., \& Gherson, T. (2009). Effect of priming cooperation or individualism on a collective and interdependent task: Changeover speed in the $4 \mathrm{x}$ 100-meter relay race. Journal of Sport and Exercise Psychology, 31, 380-389

Chen, M., \& Bargh, J.A. (1997). Nonconscious behavioural confirmation processes: The selffulfilling nature of automatically activated stereotypes. Journal of Experimental Social Psychology, 19, 541-560.

Collins, D., Jones, B., Fairweather, M., Doolan, S., \& Priestly, N. (2001). Examining anxiety associated changes on movement patterns. International Journal of Sport Psychology, $31,223-242$.

Dijksterhuis, A., \& Bargh, J. A. (2001). The perception-behaviour expressway: Automatic effects of social perception on social behaviour. In M. P. Zanna (Ed.), Advances in experimental social psychology (Vol. 33, pp. 1-40). San Diego, CA: Academic Press.

Dijksterhuis, A., \& Van Knippenberg, A. (1998). The relation between perception and behaviour, or how to win a game of Trivial Pursuit. Journal of Personality and Social Psychology, 75, 862-871.

Fitts, P.M., \& Posner, M.I. (1967). Human Performance. Belmont, CA: Brooks/Cole.

Gray, R. (2004). Attending to the execution of a complex sensorimotor motor skill: Expertise differences, choking, and slumps. Journal of Experimental Psychology: Applied, 10, 42-54.

Gucciardi, D. F., \& Dimmock, J. A. (2008). Choking under pressure in sensorimotor skills: Conscious processing or depleted attentional resources? Psychology of Sport and Exercise, 9, 45-59. 
Hardy, L., Mullen, R., \& Jones, G. (1996). Knowledge and conscious control of motor actions under stress. British Journal of Psychology, 87, 621-636.

498

499

Hardy, L., Mullen, R., \& Martin, N. (2001). Effect of task-relevant cues and state anxiety on motor performance. Perceptual and Motor Skills, 92, 943-946.

Higgins, E.T., Bargh, J.A., \& Lombardi, W. (1985). The nature of priming effects on categorization. Journal of Experimental Psychology: Learning, Memory, and Cognition, 11, 59-69.

Hull, J.G., Stone, L.B., Meteyer, K.B., \& Matthews, A.R. (2002). The nonconsiousness of consciousness. Journal of Personality and Social Psychology, 83, 406-424.

Jackson, R.C., Ashford, K.J., \& Norsworthy, G. (2006). Attentional focus, dispositional reinvestment and skilled motor performance under pressure. Journal of Sport and Exercise Psychology, 28, 49-68.

Macrae, N.C., Bodenhausen, G.V., Milne, A.B., Castelli, L., Schloercheidt, A.M., \& Greco, S. (1998). On activating exemplars. Journal of Experimental Social Psychology, 34, $330-354$.

Masters, R.S.W. (1992). Knowledge, nerves and know-how: The role of explicit versus implicit knowledge in the breakdown of a complex motor skill under pressure. British Journal of Psychology, 83, 343-358.

Masters, R.S.W., \& Maxwell, J. (2008). The theory of reinvestment. International Review of Sport and Exercise Psychology, 1, $160-183$.

Merikle, P.M., \& Daneman, M. (1996). Memory for unconsciously perceived events: Evidence from anesthetized patients. Consciousness and Cognition, 5, 525-541.

Merikle, P.M., \& Daneman, M. (1998). Psychological investigations of unconscious perception. Journal of Consciousness Studies, 5, 5-18. 
Mesagno, C., Marchant, D., \& Morris, T. (2008). A pre-performance routine to alleviate choking in "choking-susceptible" athletes. The Sport Psychologist, 22, 439-457.

Mesagno, C., \& Mullane-Grant, T. (2010). A comparison of different pre-performance routines as possible choking interventions. Journal of Applied Sport Psychology, 22, 343-360.

Mullen, R., \& Hardy, L. (2000). State anxiety and motor performance: Testing the conscious processing hypothesis. Journal of Sports Sciences, 18, 785-799.

Mullen, R., \& Hardy, L. (2010). Conscious processing and the process goal paradox. Journal of Sport and Exercise Psychology, 32, 275-297.

Mullen, R., Hardy, L., Tattersall, A. (2005). The effects of anxiety on motor performance: a test of the conscious processing hypothesis. Journal of Sport and Exercise Psychology, 27, 212-225.

Nelson, L.D., \& Norton, M.I. (2005). Form student to superhero: Situational primes shape future helping. Journal of Experimental Social Psychology, 41, 423-430.

Pijpers, J.R., Oudejans, R.R.D., Holsheimer, F., \& Bakker, F.C. (2003). Anxietyperformance relationships in climbing: A process-oriented approach. Psychology of Sport and Exercise, 4, 283-304.

Srull, T.K., \& Wyer, R.S. (1979). The role of category accessibility in the interpretation of information about persons: Some determinants and implications. Journal of Personality and Social Psychology, 10, 1660-1672.

Stone, J., Lynch, C.I., Sjomeling, M., \& Darley, J.M. (1999). Stereotype threat effects on black and white athletic performance. Journal of Personality and Social Psychology, $77,1213-1227$. 
543 Tipper, S.P., \& Weaver, B. (1998). The medium of attention: Location-based, object-centered or scene-based? In R. Wright (Ed.), Visual attention (pp. 77-107). New York: Oxford University Press.

546 Vine, S.J., Moore, L.J., \& Wilson, M.R. (2011). Quiet eye training facilitates competitive putting performance in elite golfers. Frontiers in Psychology, 2, 8.

548 Wheeler, S.C., \& Petty, R.E. (2001). The effects of stereotype activation on behaviour: A review of possible mechanisms. Psychological Bulletin, 127, 797-826.

550 Winter, S., \& Collins, D. (2013). Does priming really put the gloss on performance? Journal of Sport \& Exercise Psychology, 35, 299-307.

Wulf, G. (2013). Attentional focus and motor learning: a review of 15 years. International Review of Sport and Exercise Psychology, 6, 77-104.

554 Wulf, G., McNevin, N.H., \& Shea, C.H. (2001). The automaticity of complex motor skill learning as a function of attentional focus. The Quarterly Journal of Experimental Psychology, 54A, 1143- 1154

Zachry, T., Wulf, G., Mercer, J., \& Bezodis, N. (2005). Increased movement accuracy and reduced EMG activity as the result of adopting an external focus of attention. Brain Research Bulletin, 67, 304-309. 

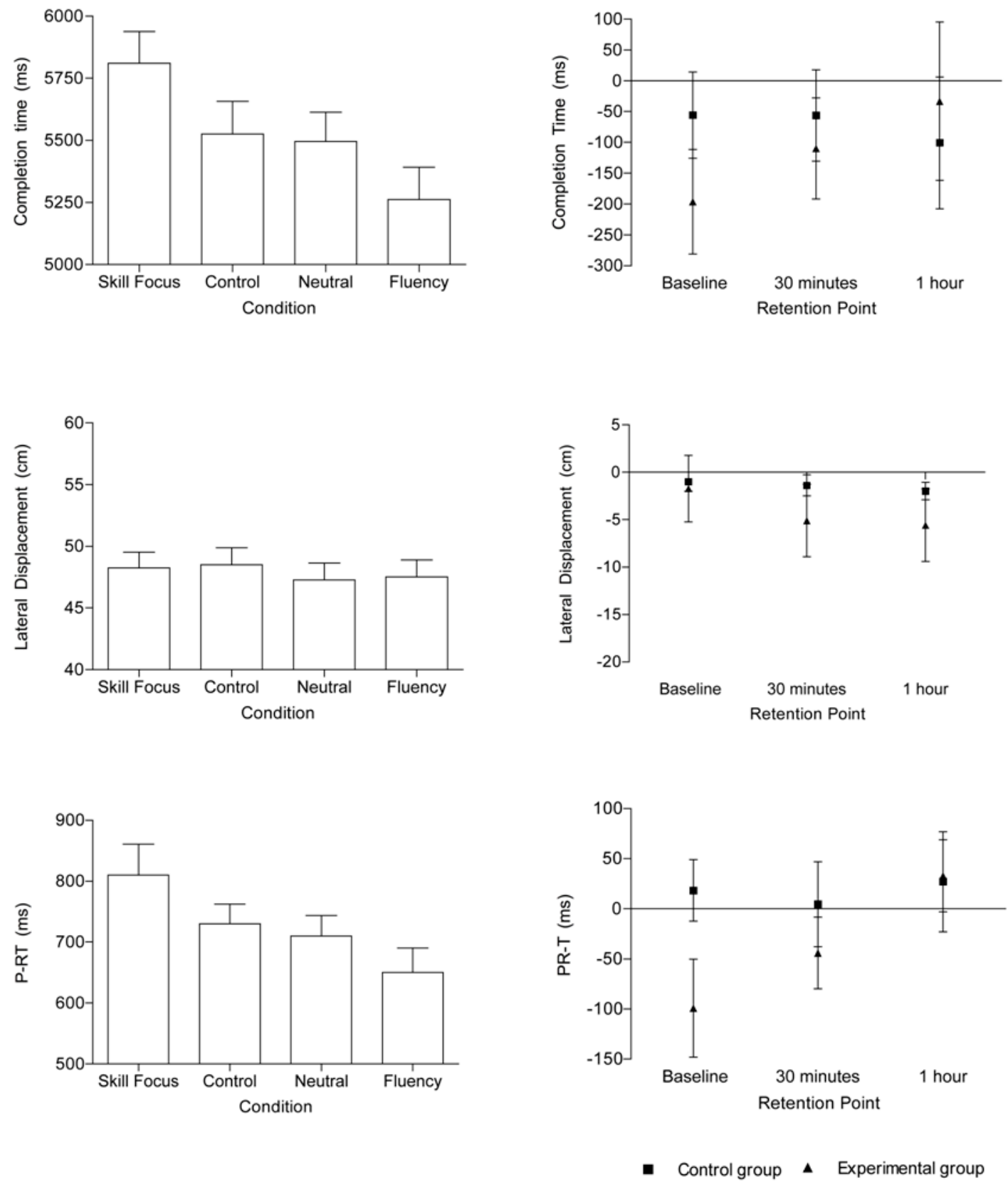

563

564 Figure 1. Mean $( \pm \mathrm{SE})$ trial completion time, lateral displacement and probe-reaction time $(\mathrm{P}-$

565 RT) under priming and control conditions (left pane) and during the retention period (right

566 pane). 
${ }^{1}$ Separate 2 (trial type: with secondary task/without secondary task) x 4 (condition: fluency/skill-focus/neutral/control) repeated measures ANOVAs, were conducted with task completion time and lateral displacement serving as dependent variables, to confirm that the dribbling task performance was not affected by the P-RT task. For task completion time, the analysis revealed a non-significant main effect for trial type, $F(1,23)=.47, p=.50, \eta_{p}{ }^{2}=$ .02 , and a non-significant trial type $\mathrm{x}$ condition interaction, $F(3,21)=1.70, p=.20, \eta_{p}{ }^{2}=$ .20. Lateral displacement analysis revealed a non-significant main effect for trial type, $F(1$, $23)=3.31, p=.08, \eta_{p}{ }^{2}=.13$, and a non-significant trial type $\mathrm{x}$ condition interaction, $F(3$, $21)=.49, p=.69, \eta_{p}{ }^{2}=.07$. These result indicate that the dependent variables were unaffected by completion of the secondary task.

${ }^{2}$ One-way repeated measures ANOVAs were conducted for each condition with trial number serving as the repeated measure and completion time, lateral displacement and P-RT serving as the dependent variables. For all conditions and variables, non-significant results were observed indicating the maintenance of a prime effect across trials. Time: Fluency: Wilks' Lambda $=.79, F(4,20)=1.30, p>.05, \eta_{p}{ }^{2}=.21$; Skill focus: Wilks' Lambda $=.83, F(4,20)$ $=1.03, p>.05, \eta_{p}{ }^{2}=.17 ;$ Neutral: Wilks' Lambda $=.78, F(4,20)=1.41, p>.05, \eta_{p}{ }^{2}=.22 ;$ Control: Wilks' Lambda $=.74, F(4,20)=1.75, p>.05, \eta_{p}{ }^{2}=.26$. Lateral Displacement: Fluency: Wilks' Lambda $=.91, F(4,20)=.51, p>.05, \eta_{p}{ }^{2}=.09$; Skill focus: Wilks' Lambda $=.79, F(4,20)=1.35, p>.05, \eta_{p}{ }^{2}=.21$; Neutral: Wilks' Lambda $=.84, F(4,20)=.97, p>.05$, $\eta_{p}{ }^{2}=.16$; Control: Wilks' Lambda $=.87, F(4,20)=.73, p>.05, \eta_{p}{ }^{2}=.13$. P-RT: Fluency: Wilks' Lambda $=.88, F(3,18)=.85, p>.05, \eta_{p}{ }^{2}=.12$; Skill focus: Wilks' Lambda $=.97$, $F(3,18)=.18, p>.05, \eta_{p}{ }^{2}=.03$; Neutral: Wilks' Lambda $=.81, F(3,18)=1.44, p>.05, \eta_{p}{ }^{2}=$ .19; Control: Wilks' Lambda $=.94, F(3,18)=.41, p>.05, \eta_{p}{ }^{2}=.06$. 